\title{
LAS FORMAS SENSORIALES EN EL CASO MÁS VIDA GUADALAJARA: ANÁLISIS A PARTIR DE BIRGIT MEYER ${ }^{1}$
}

\section{Edson Fernando Gomes ${ }^{2}$}

Resumen: Este artículo se desprende de la investigación de maestría titulada "Cuando la fe se vuelve marca: la comunicación organizacional y el cristianismo emergente en el caso 'Más Vida Guadalajara”, se desarrolló con la finalidad de comprender y explicar cómo intervienen las estrategias de comunicación en iglesias del cristianismo emergente, como el caso de Más Vida Guadalajara. La religiosidad que se practica, se reproduce y enseña en esta iglesia utiliza formas sensoriales (Meyer, 2018) como herramienta para captar la atención de los participantes en sus reuniones y así generar una experiencia, por medio de ella se transmite un sentido a las y los participantes. Este artículo pretende analizar estas formas sensoriales identificadas en el caso Más Vida Guadalajara, a través de la visión de Birgit Meyer sobre la estética de la persuasión y las formas sensoriales.

Palabras-clave: Más Vida; Formas Sensoriales; Estética de la Persuación; Birgit Meyer.

\section{SENSORIAL FORMS IN THE MÁS VIDA GUADALAJARA CASE: ANALYSIS} FROM BIRGIT MEYER

\begin{abstract}
This article is derived from the master's research entitled "Cuando la fe se vuelve marca: la comunicación organizacional y el cristianismo emergente en el caso "Más Vida Guadalajara" that sought to understand and explain how communication strategies intervene in emerging Christian churches such as the case of Más Vida Guadalajara. The religiosity that is practiced, reproduced, and taught in this church uses sensory forms (Meyer, 2018), as a tool to capture the attention of the participants of its meetings and thus generate an experience, and through it

${ }^{1}$ Como citar: GOMES, Edson Fernando. Las formas sensoriales en el caso Más Vida Guadalajara. Debates do NER, Porto alegre, ano 21, n. 39, p. 293-316, 2021.

${ }^{2}$ Mestre em Comunicação da Ciência e da Cultura pelo Instituto Tecnológico y de Estudios Superiores de Occidente da Universidad Jesuita de Guadalajara, México. E-mail: edsonbr. mex@gmail.com. ORCID: https://orcid.org/0000-0003-4726-2684.
\end{abstract}


transmit a meaning these participants. This article aims to analyze these sensory forms seen in the Más Vida Guadalajara case, through the lens of Birgit Meyer's work on the aesthetics of persuasion and the sensory forms.

Keywords: Más Vida; Sensorial Forms; Aesthetics of Persuasion; Birgit Meyer.

\section{CONTEXTUALIZACIÓN}

Este artículo se desprende de una investigación de maestría que tenía la finalidad de comprender y explicar cómo intervienen las estrategias de comunicación en iglesias del cristianismo emergente a partir del caso Más Vida Guadalajara. Derivado de observación, entrevistas y análisis crítico de los resultados del trabajo de campo, en dicha investigación (Gomes, 2020b) se comprobó que la religiosidad que se practica, reproduce y enseña en estas iglesias utiliza formas sensoriales (Meyer, 2018) como herramientas para captar la atención de las y los participantes en sus reuniones. De esta manera se genera lo que el autor conceptuliza como "Experiencia Más Vida", la iglesia transmite un sentido a quienes participan en las reuniones y viven esta experiencia. En este artículo se busca analizar estas formas sensoriales del caso Más Vida Guadalajara, a través de los lentes del trabajo de Birgit Meyer (2018).

Más Vida Guadalajara es uno de los campus de la iglesia cristiana mexicana Más Vida, esta iglesia se nació en la ciudad de Morelia en el estado de Michoacán, donde hasta el dia de hoy tiene su sede. Su pastor senior es Andrés Spyker, quien es una figura reconocida en el cristianismo latinoamericano contemporáneo. Esta iglesia destaca por su estilo estético secular, su producción musical, las predicaciones de su pastor, la práctica rutinaria de transmitir videos de predicaciones de Andrés Spyker los domingos y por no tener un predicador en vivo, solamente un pastor de campus que se dedica a dirigir la presentación del evento (Gomes, 2020b). 
Más Vida es una iglesia cristiana con características de iglesia postdenominacional, en el trabajo de Ibarra (2019) encontramos una explicación de este tipo de iglesia y su diferencia con una iglesia no-denominacional.

El cristianismo no-denominacional hace referencia a todas aquellas congregaciones que prefieren abstenerse de pertenecer - generalmente de forma explícita - a iglesias protestantes de primera línea, o a grandes asociaciones pentecostales — como a Asambleas de Dios—, en ese sentido, no es extraño que exista una correlación entre algunos grupos evangélico-pentecostales y lo no-denominacional. La autonomía, y la libertad que ésta conlleva es importante para estos grupos, pues a partir de ello pueden conducir ministerios a su gusto sin ningún tipo de interferencia externa; (...) Por otra parte, lo post-denominacional se diferencia de lo no-denominacional porque, en general, asume que la verdadera Iglesia de Cristo puede coexistir entre diferentes denominaciones y grupos cristianos, sin estar restringida al grupo religioso de uno mismo (...) lo post-denominacional tiene un componente individualista más desarrollado que lo no-denominacional; a pesar de ello, esta componente individualista no es final, es un detonador que lleva a las personas a desertar de organizaciones religiosas a las que pertenecían por una disonancia cognitiva entre lo predicado por el grupo y lo entendido y esperado por la persona (Placentra Johnston, 2012). Es así como los grupos post- denominacionales se conforman de personas que dejaron de asistir a sus congregaciones pentecostales, neopentecostales o no-denominacionales porque no respondían a sus exigencias y expectativas cognitivo-culturales y trascendentales (Ibarra, 2019, p. 98-99).

Estas iglesias son una tendencia, no poseen ningún tipo de relación insitucional con otras denominaciones, son iglesias independientes con prácticas racionales y sensoriales. Particularmente, presentan un alto nivel de secularización y centralidad en la producción musical con estándares seculares, producción cultural y mezcla constante con la cultura pop sin intenciones de cambiar la cultura sino de estar presentes en ella y ser relevantes para el público de su época. Al hablar de estándares seculares, resulta importante aclarar que en Más Vida se observa la intención de promover 
una religiosidad individualizada y a su vez enfocada en el convivio dentro de una comunidad que busca vivir el cristianismo protestante a través de un acercamiento con las esferas seculares y culturales del mundo actual. (Ibarra, 2019; Gomes, 2020b).

Iglesias postdenominacionales, como Más Vida Guadalajara son parte del movimiento del cristianismo emergente (Ibarra, 2021), utilizan la producción discursiva y musical religiosa de acuerdo con estándares seculares para generar emociones en quienes participan en sus rituales, esta experiencia se adecua a lo que su público busca y encuentra atractivo como experiencia espiritual o religiosa. Las formas sensoriales religiosas tienen gran notoriedad en la religiosidad vivida en estas iglesias representando aspectos sensoriales, estéticos y artísticos que influyen en la experiencia, identificación y permanencia de las personas participantes en las reuniones de Más Vida Guadalajara.

El cristianismo emergente (Ibarra, 2019) se caracteriza por las redes de apoyo entre iglesias pertenecientes al mismo movimiento independiente, dichas iglesias tienen público y religiosidad en común. Se unen elementos estéticos, artísticos y simbólicos de la cultura pop conectándose con los millennials ${ }^{3}$ y se enfocan en una población de clase media, media alta y

${ }^{3}$ De acuerdo con Ibarra (2019) página 57: “...el movimiento cristiano emergente es liderado por millennials, es decir, personas que nacieron entre 1981 y 1996 de acuerdo con la definición más reciente del Pew Research Center. Hablar de los sujetos de estudio de esta investigación es hablar de personas que cruzaron el umbral hacia la mayoría de edad cuando ocurría la explosión comunicativa provocada por la internet, es hablar de personas que llevaron a cabo los procesos de adaptación más intensivos en cuestión de entretenimiento y comunicación, a diferencia de los post-millennials, quienes nacieron inmersos en estas dinámicas que abrieron posibilidades y ventanas a la diversidad en toda su extensión: artística, cultural, religiosa, política, etcétera.” “...entender la categoría millennial de manera cultural, es decir, ligada a ciertas prácticas, así como a formas de consumo y de pensar relativamente generalizadas entre los miembros de un grupo dentro de un contexto determinado." (Ibarra. 2019, p. 57).

Debates do NER, Porto Alegre, ANo 2 I, N. 39, P. 293-3 I6, JAN./JUl. 202 I 
alta; estas iglesias son parte de una tendencia de nuevos grupos sin denominación que, por más que poseen prácticas religiosas pentecostalizadas y características doctrinales de iglesias reformadas, no caben en las categorías de iglesias históricas, pentecostales o neopentecostales.

Las praticas religiosas que envuelven formas sensoriales son fundamentales para la comprensión del fenómeno de las iglesias postdenominacionales, ya que las personas que frecuentan Más Vida afirman sentirse más atraídas a las prácticas religiosas que son evidentemente sensoriales de una manera más intensa que a prácticas no tan evidentes como el caso de iglesias protestantes históricas. De acuerdo con Meyer (2018) no existe realmente una religión que no tenga prácticas sensoriales en absoluto ya que todas las religiones envuelven estas prácticas en diferentes niveles, como podría ser incluso una oración en silencio o la lectura de la Biblia.

La atracción a prácticas sensoriales más evidentes con un expresivo tono emocional, tiene que ver con que las formas sensoriales son religiosidades que envuelven la práctica en el núcleo de una religiosidad, es la mediación entre lo divino y lo terreno, se da por medio de materialidades sensoriales, lo cual convierte la experiencia religiosa en algo más real y menos abstracto (Gomes, 2020a).

\section{FORMAS SENSORIALES Y ESTÉTICA DE LA PERSUACIÓN}

El concepto de formas sensoriales surge a partir de la necesidad de abordar la forma y la estética religiosas sin restarles valor a las mismas, así como de la investigación de la autora B. Meyer sobre el pentecostalismo en Ghana. Meyer observó cómo lo material y lo espiritual están directa e intrínsecamente relacionados en la religiosidad pentecostal, para construir el concepto forma sensorial, la autora se basó en la comprensión de la religión como una práctica de mediación entre los humanos y una divinidad. La cuestión de la mediación intuye que existe una separación o distanciamiento entre los dos puntos, por eso la necesidad de una mediación, en este 
caso a través de las formas sensoriales. La autora entiende la forma como un dispositivo necesario para expresar la doctrina, las creencias, lo dicho racional de las religiones de salvación de Weber. Las formas sensoriales son:

...modos autorizados de organizar e invocar acceso a lo transcendental que construyen el contenido (creencias, doctrinas, conjunto de símbolos) y las normas religiosas. Esas formas poseen un papel central en la modulación de los practicantes como sujetos religiosos, envolviéndolos en practicas particulares de culto y estándares de sentimiento. En consecuencia, formas sensoriales son parte de una estética religiosa específica, a la cual comanda un compromiso sensorial de los seres humanos entre sí y con lo divino, generando sensibilidades específicas (Meyer, 2018, p. 29-30).

Con lo anterior, podemos entender que las formas sensoriales son una manera de tener acceso y conexión con lo trascendental, estas formas elaboran sus propias reglas, prácticas religiosas y construyen contenidos religiosos. La mediación efectuada por las formas sensoriales define una estética religiosa comprometida con el ámbito sensorial del humano y su conexión con lo transcendental. A partir de esta definición de Meyer, podemos entender que los sujetos se sienten más atraídos por las prácticas sensoriales de una manera más intensa que a las prácticas religiosas que no envuelven cuestiones sensoriales.

La capacidad de tener acceso mental a lo divino y poder sentirlo, renueva la fe de algunos, sana dudas de otros y definitivamente cautiva a muchos. Lo sensorial hace que la creencia se vuelva un hecho, una realidad; esto le atribuye un nivel de poder a la experiencia, ya que la sensación física de que algo es de la realidad genera una intensidad y un nivel de apropiación diferente. Cuando en el pentecostalismo se enfatizan las sensaciones corporales de los sujetos como prueba que define si estos tuvieron la presencia y el poder del Espíritu Santo, se transforma el cuerpo del sujeto en algo que define tanto la verdad como la identidad. Existe una popularidad de las experiencias y sensaciones de lo sobrenatural en las comunidades religiosas protestantes, aunque el propio Weber tome estas experiencias como cuestiones 
de religiosidades mágicas. El pentecostalismo que surgió aproximadamente hace 100 años propuso una nueva forma de religiosidad y relación con Dios. La autora B. Meyer (2018) afirma que la experiencia de pentecostés es parte fundamental y central en el cristianismo ya que es el punto que marca la sustitución de Jesús Cristo por el Espíritu Santo.

Meyer (2018) denota cómo en el campo de los estudios religiosos las iglesias pentecostales se han transformado en asuntos centrales para el estudio del cristianismo. La autora afirma que el pentecostalismo proporciona, en la visión de Ruth Marshall, el "arquetipo contemporáneo del cristianismo como una 'comunidad sin una institución', más una comunidad de un nuevo tipo, propia de las formas de conexión difusas, individualizadas y no isomorfas de nuestro mundo globalizado" (Marshall, 2009, p. 208). La presencia del Espíritu Santo en el cuerpo del creyente hace de su cuerpo, en la visión de Meyer, un receptáculo del poder divino. Las experiencias afloran emociones y esa es una de las características mas prominentes de las iglesias pentecostales, su apelación sensorial. Dicha experiencia de la presencia divina en el cuerpo humano, en la visión de Meyer, necesita una cierta estética religiosa específica; no es algo que simplemente sucede de la nada mientras el sujeto está en su trabajo o haciendo otras actividades, en general esto parte de una serie de acontecimientos y de la suma de varios factores que están conectados a un ambiente, una comunidad y espacio-tiempo específico.

Meyer hace una crítica hacia la visión limitada del pentecostalismo a través del protestantismo. La autora entiende que la visión protestante concede cierto privilegio a la visión especifica de que el protestantismo es una religión racional, intelectual, que rechaza cuestiones místicas y mágicas, una religión que por ser tan racional ya trascendió la estancia del cuerpo y la necesidad de cuestiones de naturaleza sensorial. Con esto, el protestantismo rechaza el valor de lo que la autora llama "materialidades", por ejemplo la música y el cuerpo.

En "La Ética Protestante y el Espíritu del Capitalismo", Max Weber explicó cómo el protestantismo calvinista, promovió el sistema económico 
del capitalismo (Weber, 1922). A pesar de que Meyer aplaude el trabajo del clásico Weber, la autora declara que su argumento central es que la dimensión sensorial del pentecostalismo la lleva a hacer una revisión crítica de la división de Weber de las experiencias religiosas que son:

- Religiosidad Mágica, consiste en la atribución de poder a las prácticas religiosas, rituales, etc.

- Religiones de Salvación, consisten en cuestiones racionales, que dispensan rituales religiosos.

Esta distinción entre religiosidades queda explícita en el texto "Rechazos Religiosos del Mundo y Sus Direcciones" donde Weber (1948) habla sobre estética y la relación entre la religiosidad mágica y el arte. El autor entiende esta relación como la más antigua de la religión, donde la religiosidad estaba limitada a ser entendida por medio de bailes, construcciones y diversas materialidades que auxiliaban a que los sujetos la entendieran como algo real; la religiosidad mágica para Weber es estereotipada, inferior racional e intelectualmente hablando en comparación con la religiosidad de las religiones de salvación, donde se valorizan los lazos entre adeptos de la comunidad antes que la familia, la fe como aquello que no se ve antes que el placer de lo terreno. Para Weber la religiosidad que promueve un distanciamiento de lo secular (incluye el arte y la estética) es superior a la que no lo promueve, en el trabajo de Meyer encontramos una referencia a Weber para argumentar esta idea:

El arte como soporte de medios mágicos es, para la ética religiosa de la fraternidad, así como para el rigorismo apriorístico, no sólo depreciada, como se pone directamente en sospechas. Por un lado, la sublimación de la ética religiosa y la búsqueda de la salvación y, por otro lado, el desdoblamiento de la legalidad del propio arte tiende ya en sí a la conformación de una relación de tensión progresiva. Toda la religiosidad de redención sublimada tiene en vista solamente el sentido, y no la forma, de las cosas y acciones relevantes para la salvación. Ella desprecia la forma como siendo algo muy fortuito, de la criatura, carente de sentido (Weber, 1948, p. 341). 
Meyer afirma que Weber por una parte sugiere que el arte es una clase de blasfemia en potencia, a su vez el autor entiende que la relación entre arte y religión podría ser "restaurada" cuando dice: "Cuanto más desearon ser religiones de masa universalistas y fueron, por lo tanto, dirigidas a la propaganda emocional y a los llamados de masa, más sistemáticos se convirtieron en sus alianzas con el arte" (Weber, 1948, p. 343). Con lo anterior, podemos identificar como idea principal que para llegar a niveles globales, las religiones tienen que sacrificar la racionalidad religiosa y unirse más con el arte; podríamos agregar además, con materialidades sensoriales.

El deseo de masificación del cristianismo viene desde sus orígenes, ya que la misión encomendada por Jesús Cristo era la de ir a todas las naciones anunciando el evangelio. La razón por la cual existe una presencia del arte en las religiones de matriz cristiana y sobre todo en el Protestantismo posmoderno es que han encontrado en el arte la manera de conectarse con las personas de una forma efectiva y agradable para el público y las personas voluntarias. A través del arte, se le da espacio a los sujetos para que utilicen sus habilidades de forma creativa y recreativa, esto les brinda un espacio de presentación, a quienes no tienen el deseo de ser el centro de atención se les da un espectáculo. El arte y la religión poseen un potencial para aflorar experiencias, sensaciones y emociones, el teólogo alemán Friedrich Schleiermacher (Hoenderdaal, 1948, p. 81) consideró el arte y la religión como dos almas amigas, aún así veía el esteticismo todavía muy asociado al catolicismo y esto como un riesgo de que se adorara el arte sacro y a la iglesia institucional en vez de a Dios.

Meyer afirma que "las formas religiosas cargan una estética de persuasión específica, a través de la cual una presencia divina transcendental debe ser percibida como real y poderosa" (Meyer 2018, p. 27). De acuerdo con lo anterior, podemos entender que en el estudio de la religión, sobre todo de la religión protestante, la forma recibe poca atención y es vista como una distracción, este desprecio por la forma y la estética está directamente asociado a la idea weberiana de que las religiones de salvación son más elevadas intelectualmente al dejar la estética y la forma en un segundo plano, 
pues eso las aleja de lo sensorial y sentimental y las acerca a lo racional y "puro", esta idea elimina la posibilidad de una crítica de la estética y de las vías en que las formas religiosas poseen apelación y persuasión.

La crítica que Meyer señala en la visión de Weber en cuanto a que la estética y el arte son usadas para servir como propaganda emocional y llamado a las masas, frena el avance de la comprensión de este fenómeno, ya que al llevar a la estética al campo de los sentimientos irracionales y cuestionables, se pierde la noción e invitación de Meyer de analizar a la religión como propiciadora de estética. En este punto, podría concluirse que las formas sensoriales son religiosidades que tienen las prácticas sensoriales no como algo externo, sino que en el núcleo de su religiosidad la mediación entre lo divino y lo terreno se da por medio de materialidades sensoriales.

El concepto de estética de persuasión de Meyer (2018), está intrínsecamente conectado a las formas sensoriales, es una herramienta valiosa para explicar cómo la religión transforma los sentidos de los sujetos y permite formas de incorporación de lo divino por medio de formas sensoriales. La autora argumenta que la estética religiosa "autoriza una distribución específica de lo sensible que propicia la experiencia religiosa” (Meyer, 2018, p. 36), esto significa que existen sensaciones y experiencias activadas y propiciadas por la estética especifica religiosa, por lo tanto en el ambiente religioso, la iglesia y su sistema administran las sensaciones a partir de las estructuras religiosas ya constituidas y repetidas.

La estética de la persuasión es una herramienta de los religiosos para convencer acerca de su conexión con lo divino, esto influye en la permanencia religiosa de los sujetos, desde el punto de vista en que se quedan por sentir una conexión y un encuentro real con lo trascendental (Meyer, 2018, p. 37). Meyer relaciona la persuasión con la retórica, que claramente es un medio de comunicación que posee una conexión entre emisores y receptores del mensaje, entendiendo que la retórica hace uso de "estilos particulares que apelan a los sentidos e invocan emociones” (Meyer, 2018, p. 38), para así efectuar su objetivo de persuasión y convencimiento. Las formas sensoriales son la mediación persuasiva indispensable que permite 
que la relación con lo divino sea experimentada y entendida como frente real a cualquier clase de incredulidad. La autora argumenta que incluso Barth reconoció que Dios "persuade" a los seres humanos por medio de la "forma y figura" (Meyer, 2018).

Entre los argumentos de Meyer para explicar qué son las Estéticas de Persuación, se vincula directamente con esta investigación, aquél en el que menciona que debería ser obvio en el estudio del pentecostalismo la importancia de las formas sensoriales, "cuando las formas sensoriales son vistas como indispensables para evocar y mantener la experiencia religiosa, queda claro que los estilos que son parte de esas formas precisan estar en el centro de las atenciones" (Meyer, 2018, p. 38). El gran cambio que ofecen el cristianismo emergente y las Iglesias Postdenominacionales son exactamente en el estilo y en la forma en la cual es presentado el cristianismo por medio de formas sensoriales de persuasión.

Siguiendo la lógica de Meyer (Meyer, 2018, p. 39), la estética de persuasión postdenominacional y emergente se da a través de estrategias de comunicación con estándares seculares, la inmersión en la cultura pop contemporánea y la espectacularización del servicio religioso con la intencionalidad de atraer un público determinado, causar un sentimiento de identificación y con esto aumentar las probabilidades de que estos sujetos permanezcan en la iglesia.

\section{FORMAS SENSORIALES Y ESTÉTICA DE LA PERSUACIÓN EN MÁS VIDA}

Los aspectos referentes a formas sensoriales en Más Vida Guadalajara son evidentes ya que se pueden observar varios aspectos de formas estéticas y materiales religiosos que se relacionan con la religiosidad propuesta por esta iglesia. La figura central y tradicional del líder religioso cristiano no-católico, en la visión tradicional y estereotipada, es de un pastor vestido de traje o ropa formal, entre 40 a 60 años de edad, hablando desde un púlpito, 
exponiendo versículos bíblicos y un conjunto de normas de conducta ética y moral. Lo anterior de acuerdo con el trabajo de Vázquez (2015) donde encontramos un perfil tradicional de pastor protestante:

El pastor viste de traje con corbata, y nuevamente se tendrá que señalar que este atuendo habla de la importancia que tiene para los bautistas la reverencia tal como ellos la entienden, pues no sería bien visto que el pastor pasare al altar a predicar sin corbata, aun cuando no trajese ropa informal como tenis o pantalones de mezclilla (Vázquez, 2015, p. 124).

Andrés Spyker es un pastor que tiene entre 40 y 50 años, viste de forma juvenil, usualmente no usa traje o ropa formal y no usa un púlpito por lo que sus presentaciones son bajo un esquema de TED Talk, expone versículos bíblicos enfocándose más en experiencias propias con cuestiones de motivación personal, así como un estilo de Autoayuda y Coaching. Las TED Talk son eventos anuales donde algunos de los pensadores más importantes del mundo son invitados a compartir su historia y trabajo, es organizado por TED, una organización sin fines de lucro creada en 1984 por la Fundación Sapling, cuyo objetivo principal según los propios organizadores, es "difundir ideas"

El acrónimo “TED” viene de Tecnología, Entretenimiento y Diseño, que son las tres grandes áreas que dan forma al futuro de la sociedad de acuerdo con la visión de esta organización. Los TED Talk suceden en un auditorio con un fondo negro, donde el orador presenta sus ideas de una forma fresca, dinámica y didáctica ${ }^{5}$. En una entrevista realizada para la investigación, al preguntar sobre la predicación de Andrés Spyker se obtuvo la siguiente respuesta que ilustra las características de la forma de presentar de este pastor:

${ }^{4}$ https://www.tedxbarcelona.com/about_ted_x/ Recuperado el 29 de abril del 2020.

${ }^{5}$ https://smartalk.com.br/blog/ted-talks-o-que-e/\#O_que_e_o_TED_Talks Recuperado el 29 de abril del 2020. 
Me encanta la predicación, me encanta el mensaje, me encanta y creo que es algo que es por lo que las personas buscan también Más Vida es que nuestro Pastor Andrés hace el mensaje tan entendible y lo desmenuza de una manera que puedas aplicarlo a tu vida y puedas entenderlo. Y eso, la verdad, es como mi parte favorita que puedes crecer y escuchar algo, que es que lo voy a entender y que lo voy a poder aplicar a mi vida (Gabriel $)^{6}$.

Podemos entender las estrategias de comunicación como parte del proceso de comunicación de la iglesia, en este proceso, las estrategias son una herramienta que la iglesia usa para que las personas participantes conecten con el mensaje, a través de estéticas de persuación, se crean significados propios para las personas que influyen en su permanencia en la iglesia.

Uno de los puntos que Meyer clarifica, es que aunque las formas sensoriales proporcionen experiencias sentidas individualmente, estas sensaciones religiosas son producidas socialmente, por esta razón las formas sensoriales posibilitan e inducen la reproducción de experiencias específicas e individuales partiendo de una comunidad. Lo anterior ayuda a entender por qué a pesar de que la tecnología haya permitido ver la reunión religiosa individualmente desde la residencia de las personas por internet, una iglesia que tiene este servicio como Más Vida, no está vacía.

Si consideramos el hecho de la posibilidad de ver una película en el cine o en casa, observamos que el cine brinda una experiencia en cuanto a materialidades como el sonido, la pantalla, la comida, el ambiente, entre otras cosas; sin embargo estar con otras personas en el cine puede ser un factor que cambie la experiencia que se tiene de la película. Al comparar esto con un servicio religioso, notamos que no solamente las materialidades

${ }^{6}$ Gabriel (nombre ficticio) tenía 24 años al día de la entrevista, diseñador gráfico trabajaba con branding, comunicación para redes sociales y comunicación organizacional. Natural de San Luis Potosí, sus padres son pastores de una iglesia en ese estado. Ya venía de un contexto cristiano, y conoció a Más Vida al asistir a congresos de la iglesia. El día de la entrevista, tenía ya 2 años asistiendo a Más Vida y su cargo era de Coordinador del Área de Comunicación. 
mediadoras son clave para la experiencia de lo trascendental, sino que la presencia de una comunidad tiene importancia para una experiencia individual que parte de una comunidad.

En oposición a esto, en el protestantismo existe el movimiento de las personas que ya no van a templos y viven su religiosidad "a su manera". Estas personas ven videos de predicaciones en sus casas, cantan alabanzas, pueden ver un culto en YouTube, no van a una iglesia y se presentan como miembros de esa comunidad. En el caso Más Vida podría pensarse que las personas harían esto, ya que las predicaciones están disponibles en YouTube, sin embargo, existe un gran número de miembros presentes y activos en cuestiones de voluntariado en sus iglesias (Gomes, 2020b).

\section{I.I. LA EXPERIENCIA MÁS VIDA}

La reunión de Más Vida Guadalajara está organizada bajo una configuración de iglesia protestante, las características principales del culto radican en el tiempo que se le dedica a cada parte de la reunión; la relevancia de la experiencia de la alabanza; la predicación con técnicas de coaching y charlas motivacionales; las constantes demostraciones de emociones como felicidad por estar en la iglesia, lo cual es algo divertido y cool; los cultos temáticos(en cuestiones estéticas); la influencia de la cultura pop y su consumo; y el uso de la tecnología más avanzada posible en todas sus actividades.

Durante las observaciones realizadas para la investigación, estuvieron presentes la cuestión de tratar el ritual religioso como un evento de diversión y entretenimiento, así como los discursos de los miembros de la iglesia. Al preguntar sobre el ritual religioso de Más Vida y su relación con la idea de algo entretenido y divertido el Pastor Timoteo de campus de Más Vida Guadalajara afirmó lo siguiente:

...finalmente en mi experiencia es algo que me divierte demasiado, es algo que disfruto demasiado, disfruto te soy sincero más que mi trabajo, porque es algo que hay personas, de nuevo relaciones, amigos que lo hacemos juntos y nos la pasamos bien, o sea, tenemos una responsabilidad, claro, pero al final 
de cuentas es hacerlo de la mejor manera, de una manera divertida, alegre, o sea lo disfrutamos, cada persona, cada voluntario que está aquí son personas que se divierten, que están felices por hacer lo que hacen (Timoteo) ${ }^{7}$.

En ocasiones, mientras Timoteo dirige la reunión de Más Vida Guadalajara, afirma en el escenario que ir a la iglesia es divertido, e invita a que las personas lo repitan. La reunión religiosa como un evento de entretenimiento es un punto que hace de Más Vida una iglesia particularmente innovadora, pues propone semejanzas con un concierto de música secular y al mismo tiempo un club social donde las personas no solo van para ver un espectáculo, además van a participar activamente en el mismo. Los personas que son miembros de Más Vida, acuden para sentirse parte de este evento religioso que busca ser divertido, entretenido y trascendente, de esto se trata la Experiencia Más Vida, existe un alto nivel de espectacularización en estas reuniones, lo cual conlleva ciertas criticas tanto del público cristiano como del no cristiano. Acerca de estás críticas quienes fueron entrevistados de Más Vida, se mostraron muy tranquilos:

Esto no es, o sea, esto no es sólo un espectáculo, esto no es eso, o sea, mucha gente lo tacha de espectáculo, pero también queremos que entiendan que hay una generación actual que necesita un mensaje actual, siempre debe ser el mismo, el mismo mensaje, pero comunicado para lo actual para hacerlo relevante en el momento (Maria) ${ }^{8}$.

7 Timoteo (nombre ficticio) tenía 32 años al día de la entrevista, ingeniero mecánico, trabajaba en una empresa que se dedica a iluminación automotriz y es pastor de Más Vida Guadalajara. Vivía en Toluca donde empezó uno de los primeros campus de Más Vida, era parte de esa iglesia. Por cuestiones de trabajo se movió a Guadalajara y en ese mismo período de tiempo inició el campus Guadalajara, fue parte del Más Vida Guadalajara desde la planeación del campus. Al día de la entrevista, tenía 9 años en Más Vida y su cargo era de Pastor.

${ }^{8}$ María (nombre ficticio) tenía 29 años al día de la entrevista, consultora en temas de comunicación. Ya venía de un contexto cristiano, y conoció a Más Vida al asistir a congresos de la iglesia y por las redes sociales de la misma. Tenía 9 años en Más Vida al 
Al hablar sobre estas críticas con José, pastor y representante de Más Vida de forma institucional, demostró tener un punto de vista que indica que no suele lidiar con las críticas ya que está seguros del trabajo que hace sin criticar el trabajo de otros:

Realmente no lidiamos o sea, sí, no lidiamos. Entendemos qué, tenemos claro que somos controversiales en eso, pero tenemos claro el target, la meta a quien vamos dirigidos. Y lo que hacemos es siempre bendecir y amar las otras expresiones, nunca hablamos mal de nadie al contrario entendemos que no importa que denominación, que contexto, qué manera de ser iglesia si alguien está predicando a Cristo Jesús está haciendo el trabajo de Dios y todas las iglesias merecen nuestro respeto, nuestra honra, tratamos de cuidar las relaciones (José) $)^{9}$.

Al preguntar en las entrevistas sobre como se vive la experiencia Más Vida obtuve la siguiente respuesta:

...yo le quiero llamar Poderoso, o sea porque es colectivo, o sea un ambiente de fe, a lo mejor alguien puede venir como desanimado, puede venir como...sí, sin ganas, con tristeza y llega a un lugar donde...este...no sé o sea, estábamos cantando "cuánto nos ama Dios" pero esa persona no se sienta amada, pero al estar ahí en este ambiente conectado suceden cosas que es lo que buscamos un encuentro con Dios, o sea de una manera personal pero colectiva a la vez (Jonás $)^{10}$.

día de la entrevista, era encargada del área de jóvenes y apoyaba en temas de logística, en escenario y a veces predicando.

9 José (nombre ficticio) tenía 39 años al día de la entrevista, ministro de culto, de formación abogado, maneja negocios familiares en el área de construcción. Ya venía de un contexto cristiano, y conoció a Más Vida por el padre de Andrés Spyker. Tenía 15 años en Más Vida al día de la entrevista y su cargo era de Pastor del campus Cumbres (Morelia) y encargado de la supervisión de los campus en México.

10 Jonás (nombre ficticio) tenía 27 años al día de la entrevista, coordinador y profesor universitario de ingeniería. Conoció a Más Vida por una persona que lo invitó. Tenía 


\section{I.2. EMOCIONES Y ATMÓSFERA MÁS VIDA}

Uno de los pastores entrevistados afirmó lo siguiente acerca de las demostraciones de emociones en Más Vida:

La fe es como un bostezo, alguien bosteza y lo ves y te dan ganas de bostezar, la fe es como cuando vas a un estadio de fútbol y si no hay nadie haciendo ningún ruido, no hay ambiente, no hay ánimo, no hay expectativa de lo que va a suceder en el partido y de la fe es algo similar cuando hay una persona que está animada contagia a otras personas (Timoteo).

La afirmación de este pastor es muy importante para la comprensión de lo que es Más Vida, el pastor explica por medio de una metáfora con un partido de fútbol cómo funciona la reunión de Más Vida en cuestión de contagiar la fe. En Más Vida no solo se enfocan en la comunicación que va del escenario hacia el público, también lo hacen en la comunicación que esta sucediendo entre los miembros, inclusive de manera corporal.

El momento de alabanza es dónde se prepara a las personas para recibir el mensaje, además es un momento con un gran flujo de información hacia los espectadores/participantes de la reunión, este flujo de información, a diferencia de la predicación, ocurre de forma sutil por medio de la música, las personas no están atentas y preparadas como en la predicación para recibir información, están relajadas y abiertas.

Al preguntar sobre la experiencia que propicia el momento de la alabanza este entrevistado respondió lo siguiente:

...porque hay una atmósfera de adoración muy padre cuando es la alabanza y como está todo obscuro así como que te sientes con la libertad de llorar y/o alzar las manos sin que nadie te juzgue pues, y siento que...yo siento que

4 años en Más Vida al día de la entrevista y su cargo era de coordinador de Vida Kids (área infantil). 
aquí, bueno yo recomendaría que vinieran aquí a la reunión y toda esa onda (Tomás) ${ }^{11}$.

Esta es una estrategia de comunicación que permite o facilita la aceptación de la información y la mayor apertura a que este mensaje cause efectos en los sujetos. Meyer (2018) deja claro que las formas sensoriales, dónde se incluyen la música y el momento de alabanza, proporcionan experiencias con lo trascendental. Las materialidades mediadoras son clave para que la materialidad mediadora de este momento en Más Vida, envuelva los momentos de experiencia con lo trascendental y el momento de la alabanza.

Con la asistencia a las reuniones de Más Vida se observó mayor atención de varias personas participantes al momento de alabanza que al momento de la predicación, en las entrevistas se notó cómo la alabanza era la parte favorita de la reunión de algunos y el motivo por el cual decidieron quedarse, además es una de las estrategias de comunicación más efectivas de Más Vida relacionada a la permanencia de los sujetos en la iglesia. Al preguntar a uno de los pastores entrevistados si creía que las personas pudieran ir a Más Vida por la música, respondió lo siguiente:

Yo pienso que sí, o sea, yo pienso que ellos se encuentran una conexión con Dios al momento de cantar o al momento de experimentar no sólo la música, porque es el complemento de lo que ocurre en pantallas, lo que ocurre con las luces, es todo un complemento y creo que es una experiencia es el Espíritu Santo moviéndolos y tocándolos durante ese momento (Timoteo).

El tipo de música que se menciona, puede entenderse como música cristiana contemporánea o música de adoración y tiene una gama diversa de influencias de estilos musicales. Ibarra (2021) afirma que este estilo

11 Tomás (nombre ficticio) tenía 24 años al día de la entrevista, coordinador de marketing. Venía de un contexto católico, conoció a Más Vida cuando alguien lo invitó en la universidad, se quedó por la música y por sentirse amado y “apapachado". Tenía 5 años en Más Vida al día de la entrevista y su cargo era de líder de atención a voluntarios.

Debates do NER, Porto Alegre, ANo 2 I, N. 39, P. 293-3 I6, JAN./JUl. 202 I 
contemporáneo usado en iglesias como Más Vida Guadalajara podrían considerarse indie rock mezclado con millennial whoops, normalmente en el coro de la canción, y algunas veces inclusive con influencias del rap.

Aunque intentar definir lo qué es o no es indie rock es una tarea imposible similar a intentar delimitar qué es y qué no es $p o p$, para fines prácticos diremos que el indie rock, a pesar de ser capaz de agrupar a una diversidad de estilos e hibridaciones, se caracteriza por un equilibrio musical entre influencias pop, noise, punk psychedelia, post-punk e incluso country (Skancke, 2007). El millennial whoop hace referencia a un elemento melódico que ha sido utilizado de forma característica en la música pop de los 2010s; se trata de un patrón melódico que alterna entre la quinta y la tercera nota de una escala mayor y cantado en forma de "guas" y "ohs"; entre las canciones más famosas en utilizar este elemento se encuentran Ivy de Frank Ocean, Conqueror de AURORA, California Girls de Katy Perry, Monster de Imagine Dragons, Heart Attack de One Direction, Use Somebody de Kings of Leon, Maps de los Yeah Yeah Yeahs y Baby de Justin Bieber, entre otros (Metzger, 2016) (Ibarra, 2019).

En las observaciones se pudo notar que el momento de la alabanza se genera de forma intencional, de acuerdo con las entrevistas hechas, un ambiente de conexión con lo sagrado por medio de factores materiales y humanos.

Factores Materiales: la música, las luces apagadas del auditorio mezcladas con las luces del stage principal, el humo artificial;

Factores Humanos: las personas cantando y moviéndose en una danza al ritmo de la música, los ojos cerrados, el llanto de algunos, la risa de felicidad de otras personas, las manos levantadas, los saltos de algunos, los aplausos y los gritos de júbilo.

En el momento de alabanza hay una persona que dirige y guía a los otros participantes en lo que deben hacer, lo hace narrando acciones tales como levantar las manos, decir ciertas afirmaciones, cerrar los ojos, pensar en ciertos asuntos, todo esto mientras están cantando. Esta persona también 
indica si las personas deben orar, sobre qué deben orar y cómo lo deben de hacer.

\section{CONCLUSIONES}

De acuerdo con Weber (1948) el arte y la propaganda, que aquí se interpretan como estrategias de comunicación, son una herramienta para llevar religiones a niveles masificados al ser dirigidas por la "propaganda emocional y a los llamados de masa” (Weber, 1948, p. 343). Si traemos al autor a este contexto, para llegar a niveles globales, las religiones tienen que sacrificar la racionalidad, adherirse a las estrategias de comunicación y aumentar de forma general materialidades sensoriales y prácticas sensoriales. Aunado a esto y con la investigación de Meyer (2018) podemos afirmar que los sujetos se sienten más atraídos por las prácticas sensoriales más evidentes, como en el caso de prácticas pentecostales o como en Más Vida con cuestiones emocionales, estéticas y relacionadas directamente a sensaciones físicas, y de una manera más intensa que con prácticas religiosas que aparentemente no envuelvan cuestiones sensoriales, como la oración y la lectura bíblica. Al preguntar en las entrevistas sobre el estilo de la iglesia Más Vida, sus prácticas sensoriales y las relaciones de éstas con materialidades, se obtuvo la siguiente respuesta:

En la actualidad esto es la iglesia para nosotros siguen existiendo Iglesias tradicionales que es muy respetable y muchas personas van a esas iglesias, porque ahí se sienten cómodas, pero por ejemplo para nosotros el fondo negro o que todo este negro es porque precisamente tenemos luces en donde queremos crear atmósferas, este, crear sensaciones que las personas puedan disfrutar de la música y del mensaje, de lo que está sucediendo de una manera diferente y eso les atrae, hay muchas personas ya adultas que de repente no les gusta mucho pero se empiezan a sentir cómodos y ya entran, pero para un joven eso es "wow" y los logras atraer y se encuentran con Cristo Jesús así como 
te lo digo y entonces estamos logrando el objetivo por el cual tenemos las cosas así (Pedro) ${ }^{12}$.

Con lo anterior podemos observar cómo existen estrategias de comunicación relacionadas a materialidades, como el diseño de interiores de la iglesia y la búsqueda consciente por "crear atmósferas". El uso de la comunicación con base en las formas sensoriales y la estética de la persuasión (Meyer, 2019) hace de Más Vida una iglesia capaz de atraer a un público juvenil, conformado por millennials, caracterizado por buscar una religiosidad cristiana no católica y alejada de lo tradicional; no olvidemos que las referencias que estos públicos tienen respecto a lo "cool" se asemejan a las de Más Vida. Esta iglesia no se adapta para atraer un público, lo hace porque la iglesia está hecha por gente que piensa de forma parecida a este grupo (Ibarra, 2021).

La capacidad de Más Vida de proporcionar una experiencia numínica ${ }^{13}$ (Otto y Harvey, 1928) a través de lo sensorial tiene impacto en los buscadores (seekers) que desean encontrar más que una simple reunión religiosa. Las prácticas sensoriales provistas por Más Vida no están más cercanas a los sentidos humanos que a la racionalidad, lo que causa una identificación

12 Pedro (nombre ficticio) tenía 40 años al día de la entrevista, coordinador de marketing. Venía de un contexto cristiano, conoció a Más Vida por su hermano. Tenía 15 años en Más Vida al día de la entrevista y su cargo era de director creativo.

${ }^{13}$ De acuerdo con Otto, la experiencia del númen incluye el mysterium tremendum, sentimiento que evoca temblor y miedo, y el mysterium fascinans, sentimiento que evoca una sensación de atractivo deleite inmenso e inexplicable, algo que es fascinador y seductor (Otto \& Harvey, 1928). El resultado de esto de acuerdo con Ibarra (2019) "es una experiencia personal, en la que el individuo y sus sentidos son los protagonistas de este gran espectáculo fascinador, donde desfilan uno a uno estos sentimientos tan extraños, tan únicos, tan gloriosos, tan sublimes, y en el que hasta el alma más escéptica, razonable y fría se atreve a apostar al final, que esto no es todo lo que tenemos, que debe haber algo más trascendental, una "ligera sensación como de un recuerdo lejano o como si cayéramos de una gran altura”, como dice la cita de Sagan; ese taciturno reflejo de una remembranza desconocida, recuerdos vaporosos de un infinito" (Ibarra, 2019, p. 2).

Debates do NER, Porto Alegre, ANo 2 I, N. 39, P. 293-3 I 6, JAn./JUl. 202 I 
mayor con la institución es una producción de emociones, sentimientos y sensaciones dentro de una estética que recuerda lo que es definido culturalmente en la actualidad entre el público millenial como "cool".

El atractivo principal para quedarse en la iglesia Más Vida, de acuerdo con las personas entrevistadas, no es en sí la práctica sensorial, esto tiene sentido ya que, si estuvieran buscando experiencias lo más clara y evidentemente sensoriales posibles, estarían en una iglesia pentecostal o neo-pentecostal. La influencia principal en su permanencia es la identificación que este público tiene con las características estéticas de la institución, su comunicación, su música, sus prácticas religiosas sensoriales y cómo tratan a las personas que van a sus reuniones ${ }^{14}$.

De acuerdo con lo expuesto en el texto, al entender el concepto de las formas sensoriales como una forma de tener acceso y conexión con lo trascendental que elabora sus propias reglas y prácticas religiosas y construyen contenidos religiosos, se puede concluir, de la mano de Meyer (2018) que la mediación efectuada por las formas sensoriales define una estética religiosa comprometida con lo sensorial del humano y su conexión con lo transcendental. Las formas sensoriales religiosas tienen gran notoriedad en la religiosidad vivida en Más Vida, son aspectos sensoriales, estéticos y artísticos que influyen en la experiencia, identificación y permanencia de los participantes en sus reuniones. Las iglesias postdenominacionales buscan crear atmósferas para generar emociones y sensaciones en los participantes de sus reuniones. Las formas sensoriales religiosas poseen un papel fundamental en esto.

14 Los entrevistados em diversas veces utilizaron el término "apapachados" con lo que describian una forma cariñosa con la que fueron tratados en Más Vida.

Debates do NER, Porto Alegre, ANo 2 I, N. 39, P. 293-3 I6, JAn./Jul. 202 I 


\section{REFERENCIAS}

GOMES, Edson Fernando. ADN Más Vida: la identidad de una iglesia postdenominacional mexicana en crecimiento. Ciencias Sociales y Religión/ Ciências Sociais e Religiāo, Campinas, SP, v. 22, p. 1-16, 2020a.

GOMES, Edson Fernando. Cuando la fe se vuelve marca: la comunicación organizacional y el cristianismo emergente en el caso "Más Vida Guadalajara". Tesis de Maestría en Comunicación de la Ciencia y la Cultura, ITESO, Tlaquepaque, 2020b.

HOENDERDAAL, Gerrit Jan. Religieuze existentie en aesthetische aanschouwing: een studie over het misverstand omtrent het aesthetische element in Schleier-machers wezensbepaling der religie. Arnhem: Van Loghum Slaterus, 1948.

IBARRA, Carlos Samuel. Cristianismo Postdenominacional, Movimientos Emergentes y Deconstrucción Religiosa en el Norte de México. Tesis de Doctorado en Estudios Culturales, El Colegio de la Frontera Norte, Tijuana, 2019. IBARRA, Carlos Samuel. Beards, Tattoos, and Cool Kids: Lived Religion and Postdenominational Congregations in Northwestern Mexico. Int J Lat Am Relig, 2021.

MARSHALL, Ruth. Political Spiritualities: the Pentecostal Revolution in Nigeria. Chicago: University of Chicago Press, 2009.

MARTÍNEZ-CREIXELL, Federico. Los sistemas de significación como factores para la calidad del diálogo en la comunicación intercultural. Negociaciones entre empresarios mexicanos y estadounidenses en la industria turistica en Los Cabos, Baja California Sur. Tesis de maestría, Maestría en Comunicación de la Ciencia y la Cultura. Tlaquepaque, Jalisco: ITESO, 2011.

MEYER, Birgit. A estética da persuasão: as formas sensoriais do cristianismo global e do pentecostalismo. Debates do NER, Porto Alegre, ano 19, n.34, p. 13-45, 2018. 
MEYER, Birgit (Org.). Aesthetic Formations: Media, Religion, and the Senses. Basingstoke: Palgrave Macmillan, 2009.

MILLER, Donald Earl. Postdenominational Christianity in the Twenty-First Century. The Annals of the American Academy of Political and Social Science, v.558, n.1, p. 196-210. 1998.

OTTO, Rudolf; HARVEY, John W. The Idea of the Holy. London, Oxford University Press, 1928.

VÁZQUEZ, Patricio. La renovación de la música religiosa, su influencia en la expresión de la religiosidad y las estrategias de difusión y consolidación de tres iglesias bautistas en Ciudad Juárez, Chihuahua, México. Tesis de maestría en Antropología Social, Escuela de Antropología e Historia del Norte de México, México, 2015.

WEBER, Max. Economia y sociedad. Esbozo de sociología comprensiva. México: Fondo de Cultura Económica, 1964 [1922].

WEBER, Max. Religious rejections of the world and their directions. In: WEBER, Max; GERTH, H.H.; MILLS, C. Wright (eds., trans.), From Max Weber: essays in sociology, p.323-359, ANTHROPOLOGY D 10 WEB: Routledge, 1948.

Recebido em: 16/02/2021 Aprovado em: 05/04/2021 